\section{"Sparen" für die Jugend, kürzen für die Banken?}

Wer profitiert von mehr Wirtschaftlichkeit in der Jugendhilfe?

Angesichts der gewachsenen Schuldenbelastung der armen Städte steht nun die Wirtschaftlichkeit der Erbringung der „Pflicht“-Leistungen in der gesamten Verwaltung - und damit auch in der Jugendhilfe - auf dem Prüfstand. Dies hat die Gemeindeprüfungsanstalt Nordrhein-Westfalen (GPA) veranlasst, in den Jahren 2007 und 2008 „Konsolidierungspotentiale“ der 23 kreisfreien Städte $z u$ untersuchen und zu bewerten (GPA 2009). Im Bereich Jugend standen die Leistungsorganisation, -erbringung und Angebotssteuerung nach dem SGB VIII - in erster Linie die Hilfen zur Erziehung (HzE) und die Tagesbetreuung für Kinder im Mittelpunkt des interkommunalen Vergleichs mittels Benchmarking.

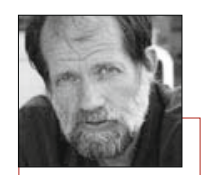

Obwohl die GPA die Städte je nach Kaufkraft, Bevölkerungsentwicklung, allgemeinen Deckungsmitteln, Arbeitsplatzzentralität, SGB II- und Arbeitslosenquoten in fünf unterschiedliche Gruppen eingeteilt hat, orientieren sich Potentiale zu größerer Wirtschaftlichkeit der Leistungen der jeweils untersuchten Stadt am Wert der Kennzahl in Dr., Diplom-Pad Leiter des RAA/Büro für interkulturelle Arbeit der Stadt Essen und langjähriger Beirat von Sozial Extra. Helmuth.Schweitzer @raa-interkulturel lesbuero.essen.de

der jeweils besten (sprich: billigsten) aller 23 Kommunen, so dass am Ende doch Äpfel mit Birnen verglichen werden.

Das größte Kürzungspotential im Bereich $\mathrm{HzE}$ wird in einer Reduktion der Anzahl der Hilfefälle je 1.000 Einwohner unter 21 Jahren, der sogenannten „Leistungsdichte“, gesehen. Dies soll durch Ausbau der präventiven Hilfen und durch gezielte Leistungssteuerung erreicht werden. Im Bereich der Kindertagesbetreuung liegt die Hoffnung auf der „Erzielung hoher Elternbeiträge zur Finanzierung der Betriebskosten“ und dem „Ausbau der Tagespflege als kostengünstige Alterna- tive zur institutionellen Betreuung“ (GPA 2009, Ju 54). Insgesamt wird auf diese Weise z.B. für die Stadt Oberhausen ein Kürzungspotential von 12 Mio Euro pro Jahrfast ein Viertel des Potentials der gesamten Verwaltung - berechnet (s. Kasten S. 28) und für Duisburg mit vergleichbarer Leistungsdichte (31 Fälle) sogar 22 Mio veranschlagt. Sind diese Erwartungen bei seit 2006 nochmals gestiegenen Fallzahlen und fehlendem qualifiziertem Personal für den wachsenden U3-Betreuungsbedarf realistisch, ohne die Standards zu senken und die Arbeitsverdichtung zu steigern? Duisburg hat 2009 insgesamt neun Mio Euro mehr ausgeben müssen, obwohl bei der HzE gekürzt wurde (NRZ vom 6. u. 11.1.11). Die Leistungsdichte erhöht sich in den armen Städten statistisch schon dadurch, dass von dort eher erziehungskompetente und sozial abgesicherte Familien ins Umland ziehen.

Als eine wesentliche Ursache für die gute Position der Stadt Gelsenkirchen im HzE-Bereich nahe der Benchmark sieht die GPA - neben dem Ausbau präventiver Hilfen - das in Fachkreisen eher kritisch beurteilte, streng zentralistische Steuerungsmodell durch den Amtslei- ter (s. Kasten S. 28). Im Gespräch mit ihm und seinem Oberhausener Amtskollegen wird deutlich, wie zwei arme Städten der Ruhrregion unter vergleichbaren Rahmenbedingungen trotz unterschiedlicher Steuerungsstruktur mit den Kürzungsvorgaben des Kämmerers bzw. der Bezirksregierung in ähnlich kreativer Form umgehen und - dank „Kevin“ - mit politischer Unterstützung sogar mehr Personal einstellen konnten. Demgegenüber zeigt der Beitrag von Ingrid Krammer und Günter Riegler aus Graz, wie in einer wirtschaftlich prosperierenden Stadt durch konsequente Umsteuerung auf eine Sozialraumbudgetierung die angestrebte Qualitätssteigerung in der Jugendhilfe mit der berechtigten Hoffnung auf geringere Kostensteigerungen verbunden wird.

\section{Literatur}

GPA NORDRHEIN-WESTFALEN (2009).

Überörtliche Prüfung kreisfreier Städte. Gesamtbericht. Herne, unveröff.

Inzwischen sind aber alle öffentlichen Haushalte (Kommune, Länder, Bund, EU, G 20) durch die Kredite und Zinszahlungen an die Banken so tief ins Netz des globalen Finanzkapitals verstrickt, dass der Wettlauf aus der Schuldenfalle der armen Kommunen in den reichen Staaten über eine Reduktion der staatlichen Daseinsvorsorge zugunsten privatwirtschaftlicher Anbieter mit staatlichen Gewinngarantien (nicht nur für die Wasserwerke!) in wachsende öffentliche und private Armut führt. Damit auf diese Weise die soziale Spaltung innerhalb der Gesellschaft nicht noch weiter gefördert wird, muss sich Jugendhilfe wieder in den lokalen Diskurs über die Frage einmischen, welche Ziele durch die Stadtpolitik vorrangig finanziert werden und ob die alten Blitzableiter in den schon absehbaren Finanzgewittern vor Ort noch funktionieren.

Abstract / Das Wichtigste in Kürze Viele Städte sind von hohen Schulden in ihren Gestaltungsspielräumen eingeengt. Kürzungen sollen Entlastung bringen, aber sind diese am Ende auch langfristig wirtschaftlich?

Keywords / Stichworte Kürzungspotentiale, Kommunen, Kennzahlen, HzE, Finanzierung. 